\title{
A smart detection system for power grounding grid based on GPR
}

\author{
Xin $\mathrm{Wu}^{1}, \mathrm{Xu} \mathrm{Zhang}^{1}$, Jianhong $\mathrm{Hao}^{1}$, Jiming Song ${ }^{2}$, Fengxian $\mathrm{Miao}^{2}$ \\ ${ }^{1}$ School of Electrical and Electronic Engineering, North China Electric Power University, Beijing, \\ 102206, China \\ ${ }^{2}$ AC Construction Branch, State Grid Corporation of China, Beijing, 100052, China
}

Keywords: Radar detection, grounding grid, lap joints, recognition.

\begin{abstract}
Grounding grid is a kind of critical infrastructure to ensure safe and stable operation of the grid and the personal safety. Buried in the ground and limited by the surface buildings, the ground grid is inconvenient to be detected and maintained. In this paper, in order to overcome the difficulties in detecting the ground grid of the high-voltage transmission and substation, we studied a detection system based on ground penetrating radar (GPR), which can present intuitive visual images of the grid, makes accurate diagnosis to the ground grid, without power failure and dug.
\end{abstract}

\section{Introduction}

The design, installation and maintenance of norms detection of grounding grid used in high voltage transmission lines and substations is the foundation to ensure the safe operation of circuits and systems, grounding grid has the characters of large-scale, complex structure and laying deep. In practical engineering, the means for measuring the grounding grid are primitive, traditional methods for measurement are indirect measurement or excavation, with blindness, and large workload, low efficiency and inadequate measurement accuracy. Meanwhile, the high-voltage transmission lines and substations is bearing the heavy responsibility of national production and people's lives, whose maintenance without power will inevitably bring many economic losses that makes the actual operation difficult. In this paper, to solve the current difficulties in the detection of ground grid used in high-voltage transmission lines and substation, we present a detection system based on ground penetrating radar (GPR), designing and forming a kind of device to the corresponding test[1-2]. Meeting the electricity system `s limitation of simple, accurate, free on-site operating conditions, the system for the grounding grid achieves diagnosis and practical detection for the grounding grid without power failure and dug [3-5]..

\section{Detection of Power Grounding grid}

\subsection{Requirement on Detection of Ground Grid}

The phenomenon in the construction of grounding grid, which do not meet the design, such as the conductor size too small, overlapping points Weld, equipment grounding, which do not comply with the original series were designed will reduce grounding grid's performance. As a result of underground operation, and harsh conditions, where it is easy to encounter moisture, soil acidity and other harmful gases, causes corrosion of the grounding grid, makes the grounding conductor or lead rust, deform, or even break, that undermines the structure initially designed, results in poor performance, threatening personal and equipment safety. It is required for the ground grid to facilitate state of the grounding grid timely and efficiently.

\subsection{Detection Methods}

The traditional measurements of grounding grid are indirect measurement or direct excavation, with blindness, and workload, slow, and poor accuracy which are limited by the field operation. Therefore, convenient, initiative and accurate measurement in grounding network testing of substation needs further development. 
Grounding resistance of grounding systems is an important technical indicator to confirm the efficacy, safety and judge the grounding system whether meets the designed parameters. Currently, measurements for earth resistance commonly are the three-pole or four-pole, frequency measurement, different frequency measurement and other traditional methods. In this paper, based on ground penetrating radar, the detection system can efficiently and accurately detect the physical state of the grounding grid, and form intuitive visual images, providing a wealth of data to ensure a timely and effective manner to achieve accurate detection of grounding grid in the ultra-high voltage transmission lines and substations. Table 1 is a comparison between the measurement based on GPR and the traditional measurement.

Table 1: Comparison of GPR detection and the traditional measurement method

\begin{tabular}{lll}
\hline Measurement methods & $\begin{array}{l}\text { ultra-high voltage } \\
\text { transmission lines and } \\
\text { substations }\end{array}$ & $\begin{array}{l}\text { the performance of ground-penetrating } \\
\text { radar detection system }\end{array}$ \\
\hline Measurement & $\begin{array}{l}\text { Excavation measurement or } \\
\text { indirect measurement }\end{array}$ & No excavation, signal penetration \\
Range & $\begin{array}{l}\text { Strong blindness } \\
\text { Workload }\end{array}$ & $\begin{array}{l}\text { Large measuring range } \\
\text { Workload }\end{array}$ \\
Efficiency & $\begin{array}{l}\text { Inefficient } \\
\text { Strong sidedness, poor }\end{array}$ & $\begin{array}{l}\text { High efficiency } \\
\text { Rich information, high precision }\end{array}$ \\
& accuracy & \\
\hline
\end{tabular}

\section{Radar Detection System Design}

\subsection{Principle of radar detection}

GPR is an active electromagnetic equipment. The basic principle is to launch a high-frequency electromagnetic waves into the ground, and then receive the reflected echo signal from the underground media [6-7]. Through analysis of the echo signal, GPR studies the characteristics of underground targets. GPR's role in the media is equivalent to a complex filter [8]. The varying absorption of electromagnetic waves and inhomogeneous properties of the medium, make physical information of different underground targets combine, when electromagnetic pulses emitted by GPR arrive receiving antenna, resulting in amplitude decreases, the frequency decreases, the phase and the reflection time change, the waveform becomes a greater difference with the original transmitted waveform, i.e., when electromagnetic wave propagates in the medium. its path, electromagnetic field strength and the waveform will vary with the changes of the medium's electrical properties and geometry changes. Thus, according to the received wave`s travel time (also known as the two-way travel time), the amplitude and waveform data can be accurately obtain the physical structure of the medium. Fig.1 shows the acquisition process of ground penetrating radar target information systems.

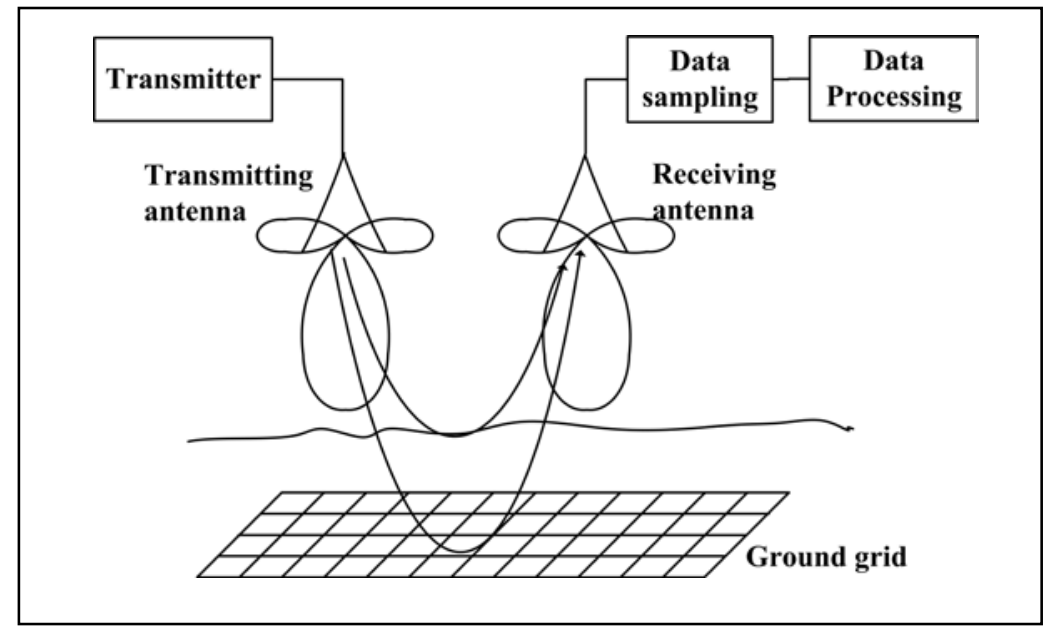

Fig.1: Information acquisition process of GPR 
The use of GPR's polarization technology could achieve accurate detection on conductors corrosion. Polarization characteristic of the radar echo is the fourth feature besides amplitude, phase and Doppler shift, used for the fine characterization of target, accurately determining the extent of corrosion of ground grid, focusing on extracting surface roughness of target, material composition and other properties. Therefore, the use of electromagnetic wave polarization characteristics can achieve the goal of characterizing the physical details of the target. Grounding conductor will be brittle, from the floor, loose, thinning, smaller or break other changes after being corroded, presenting a significant difference in polarization characteristics. Through different forms of objective circumstances varied with variable polarization polarized waves, building the corresponding database of different features, forming target recognition based on the details, we are able to accurately determine the extent of grounding grid`s corrosion.

\subsection{Parameters for GPR detection.}

According to the characteristics of GPR system, we can effectively and easily detect the physical state of grounding grid using in high-voltage transmission line. i) The GPR system can accurately position the grounding conductor and lead, testing material and shape of the ground device. ii) The GPR system could accurately detect corrosion status of grounding conductor and accurately determine location and type of faults. iii) The GPR system could accurately detect the quality and state of grounding equipment`s solder joints, effectively detect lap width and weld quality..

\subsubsection{Grounding grid parameters}

According to "National Grid substation ground grid design specifications", the material of substation ground grid is divided into two types:The first one is made in flat steel, round steel and angle iron, which the round steel is almost galvanized steel bar based mainly $\Phi 12$, Ф16, Ф18, Ф20 and other specifications; galvanized flat steel mainly for flat steel, with its width of $20 \mathrm{~mm}, 30 \mathrm{~mm}$, $40 \mathrm{~mm}$ and other specifications; angle iron are made of galvanized angle, with the specifications of the $\angle 25 \times 4, \angle 40 \times 4, \angle 50 \times 5$. The network in the process of laying grid are interconnected by ordinary welding, with poor corrosion resistance, low construction cost, and the area is large. Its service life is generally last 4-10 years. Table 2 is for the design materials of flat ground grid.

Table 2: Design materials of flat ground grid

\begin{tabular}{|c|c|c|c|c|}
\hline No. & Name & & $\begin{array}{l}\text { Model } \\
\text { specification }\end{array}$ & Remark \\
\hline 1 & Grounding & & L50入5, L2500 & Total 340m \\
\hline 2 & $\begin{array}{l}\text { Galvanized } \\
\text { steel }\end{array}$ & flat & $-80 \backslash 88$ & $\begin{array}{l}\text { Equipment } \\
\text { deflectors }\end{array}$ \\
\hline 3 & $\begin{array}{l}\text { Galvanized } \\
\text { steel }\end{array}$ & flat & $-60 \times 8$ & Level ground wire \\
\hline 4 & $\begin{array}{l}\text { Galvanized } \\
\text { steel }\end{array}$ & flat & $-50 \times 6$ & $\begin{array}{l}\text { Cable } \\
\text { indoor }\end{array}$ \\
\hline 5 & Fastener & & $\begin{array}{l}2 \times(\mathrm{M} 16 \times 35) G B 5- \\
76\end{array}$ & \\
\hline 6 & Bronze medal & & $-25 \times 4$ & \\
\hline
\end{tabular}

The second category, mainly made in Copper plated steel, copper trips, copper belt, The main specifications for copper grounding grid 's section deflectors is $200 \mathrm{~mm} 2$, and whose horizontal cross-section is $150 \mathrm{~mm} 2$. Connection method of the grounding grid is mainly exothermic welding, with high project costs and good corrosion resistance. Its service life is generally 30-60 years. Table 3 takes the main specifications of copper grounding grid. 
Table 3: Copper ground grid design of materials

\begin{tabular}{|c|c|c|c|}
\hline No. & Name & Model specification & Remark \\
\hline 1 & Grounding & $\begin{array}{l}\text { Copper rods } \Phi 14.2 \\
\mathrm{~L}=2400\end{array}$ & \\
\hline 2 & Copper bars & $\begin{array}{l}\text { Sectional } \\
\text { Area } 150 \mathrm{~mm}^{2}\end{array}$ & $\begin{array}{l}\text { Equipment } \\
\text { deflectors }\end{array}$ \\
\hline 3 & Copper bars & $\begin{array}{l}\text { Sectional } \\
\text { Area } 120 \mathrm{~mm}^{2}\end{array}$ & Level ground wire \\
\hline 4 & Copper bars & $-25 \times 4$ & $\begin{array}{l}\text { Cable } \\
\text { indoor }\end{array}$ \\
\hline 5 & Fastener & $\begin{array}{l}2 \times(\mathrm{M} 16 \times 35) G B 5- \\
76\end{array}$ & \\
\hline 6 & Bronze medal & $-25 \times 4$ & \\
\hline
\end{tabular}

\subsubsection{Functions of GPR}

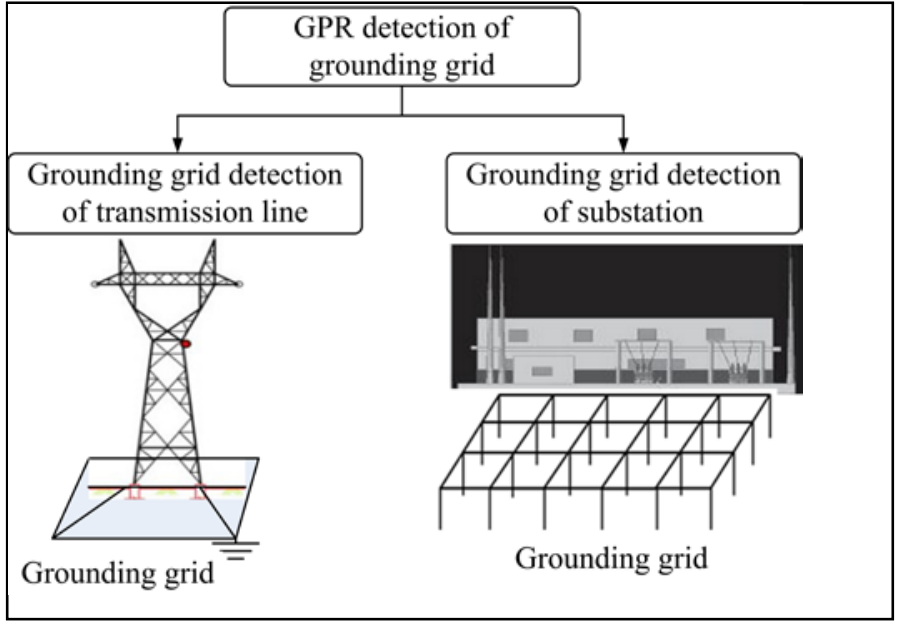

Fig.2: Detection scene of the GPR smart device

Fig.2 shows that the main use of GPR in the detection of ground grid .In the detection of highvoltage substation`s ground grid, specific functions of the GPR system are as follows: i) The devices are required to achieve accurately positioning grounding conductor and moldings, access to its laying depth and contours. ii) The GPR system can accurately detect the material and shape of the grid, with the expected resolution achieving millimeter. In practice, some parts of ground grid are made of copper material, and some parts are made of steel. The GPR can accurately detect the actual composition of the various parts. iii) The GPR can be used to determine the corrosion, breakage of ground conductor and the like. iv) It can accurately detect the situation and the quality of ground device`s solder joints, lap width and weld quality between the two parts. v) GPR can effectively determine the actual state of the underground grounding grid, such as single ground, double ground, independent grounding. vi) GPR can used to the special test for GIS and other master, including equipment grounding materials, grounding methods and other physical characteristics.

\subsection{Key technologies of radar detection of ground grid .}

\subsubsection{Realization in depth of detection}

As grounding grid is generally buried in the depth of no more than 0.8 meters, it is necessary to make some restrictions to the depth of probing depth of GPR. Because the similar principle between GPR and sounding radar, it can analogy sounding radar`s formula to that of GPR, the formula is shown as (1). 


$$
P_{R}=\frac{P_{T} G^{2} \lambda^{2} S}{(4 \pi)^{3} R^{4}} L_{01} L_{10} L_{S} \exp (-4 \alpha R)
$$

where $P_{R}$ denotes received power of GPR, $P_{T}$ is the radar's transmitter power, $G$ is the antenna gain, $S$ is the target's scattering cross section, $L_{01}$ and $L_{10}$ represent the coupling losses between receiving antenna and transmitting antenna with the ground, $R$ denotes the depth of target, $\alpha$ is integrated decay rate due to the soil absorption, $L_{\mathrm{S}}$ denotes the scattering loss of transmission medium, and $\lambda$ is wavelength.

From (1), we can draw that the detection depth of GPR is related to electromagnetic properties of local earth where the ground grid buried, transmitting frequency and transceiver`s power and receiver`s power of the radar antenna, scattering cross-sectional area of the target and the bump degree of surface. The ground penetrating depth of GPR is inversely proportional to the antenna frequency, which is also inversely proportional to the integrated decay rate reflecting the electromagnetic properties of the soil. Ground penetrating depth of GPR with frequency of $100 \mathrm{M}$ is given by Table 4.

Table 4: Depth gauge of GPR in 100M transmitting frequency

\begin{tabular}{ll}
\hline Soil type & Probing depth (m) \\
\hline Coarse sand (dry) & $25-40$ \\
Pebble & $10-15$ \\
Sand (dry) & $15-30$ \\
Sand (wet) & $5-10$ \\
Silt (dry) & $5-10$ \\
Silt (wet) & $3-8$ \\
Farming soil (dry) & $10-20$ \\
Farming soil (wet) & $5-10$ \\
\hline
\end{tabular}

\subsubsection{The realization of detection accuracy}

In the test of UHV substation's grounding grid, ground material has been given. Contrast to the large area of grounding grid, the cross-sectional area of the network's material is too small, therefore, the GPR is required for high detection accuracy.

Vertical accuracy is mainly used to detect the depth of ground grid, depending on the radar's ability to distinguish several echoes of similar time, with the time interval expressed by the following formula:

$$
\Delta t=\frac{1}{\Delta f}
$$

where $\Delta f$ is the difference between the received signal spectrum, so depth can be represented as (3).

$$
\Delta h=\frac{\Delta t v}{2}=\frac{v}{2 \Delta f}
$$

From (2) and (3), the vertical accuracy of GPR depends on propagation velocity of electromagnetic wave in the soil $\mathrm{v}$, and $\mathrm{v}$ in turn depends on the dielectric constant of the soil as well as the transmission frequency of the radar's antenna, therefore, the higher transmission frequency of the radar antenna and the larger dielectric constant of the propagation medium, result in higher the vertical accuracy. $\Delta \mathrm{f}$ relates to antenna, which is also effected by the propagation medium: a pulse wave is propagating in the ground, along with the dispersion of medium, which makes the effective bandwidth $\Delta$ f become increasingly smaller. For the vertical accuracy of GPR, the probing depth and detection accuracy is a pair of conflicting parameters, which in the practical applications must be fully weighed. The GPR in testing applications of UHV Substation`s network, due to the net is buried shallow, will not consider the vertical accuracy. Table 5 shows the detection accuracy and relative permittivity of common mediums.

Table 5: Detection accuracy and relative permittivity of common mediums 


\begin{tabular}{|c|c|c|c|c|c|}
\hline \multirow[t]{2}{*}{ Medium } & \multirow[t]{2}{*}{$\begin{array}{l}\text { Relative } \\
\text { permittivity }\end{array}$} & \multirow[t]{2}{*}{$\begin{array}{l}\text { Velocity } \\
\left(\mathrm{m} \cdot \mathrm{ns}^{-1}\right)\end{array}$} & \multicolumn{2}{|c|}{$\begin{array}{l}\text { The accuracy } \\
\text { antenna(m) }\end{array}$} & the \\
\hline & & & 35 & 100 & 200 \\
\hline Water & 81 & 0.033 & 0.24 & 0.08 & 0.01 \\
\hline Dry clay & 4 & 0.15 & 1.07 & 0.38 & 0.05 \\
\hline Wet clay & 27 & 0.05 & 0.36 & 0.13 & 002 \\
\hline Dry sand & 4 & 0.15 & 1.07 & 0.38 & 0.05 \\
\hline Wet sand & 6 & 0.12 & 0.86 & 0.30 & 0.04 \\
\hline Saturated Sand & 25 & 0.07 & 0.50 & 0.18 & 0.02 \\
\hline Dry gravel & 5.5 & 0.13 & 0.93 & 0.33 & 0.04 \\
\hline Wet gravel & 5 & 0.14 & 1 & 0.35 & 0.05 \\
\hline Limestone & 5.5 & 0.13 & 0.93 & 0.33 & 0.04 \\
\hline Organic soil & 64 & 0.04 & 0.29 & 0.10 & 0.02 \\
\hline
\end{tabular}

The horizontal precision accuracy is mainly used to detect the corrosion of the mesh material, as the parameter also depends on the ability to distinguish several echoes of similar times, which similar to the vertical accuracy.

\section{Conclusions}

The detection for the grounding grid of High-voltage line and high-voltage substation are suitable for using radar detection technology that can effectively and easily detect the physical state of the grounding grid, including the ground grid's location, depth, shape, detail, degree of corrosion. However, the difference in interference of signal and geometry between the two scenarios makes a difference in antenna technology and signal processing technology. There are more parameters for test of the substation grounding, more complex considerations, more stringent functional requirements for the equipment.

\section{Acknowledgements}

This work is supported by the research of grounding grid radar detection system for $1000 \mathrm{kV}$ UHV AC Huainan-Nanjing-Shanghai project, the National Natural Science Foundation of China under grant No. 51407072, and the Fundamental Research Funds for the Central Universities of China under grant No. 13QN01.

\section{References}

[1] Zhao,Q.Q.Lei,W,Deng,Y. Selection of GPR Antenna Frequency in Engineering Exploration, Investigation Science and Technology, 2013, (2).

[2] Lee,T. Design, implementation, optimization of GPR antenna system”, Wuhan University, 2004. In Chinese

[3] Feng,H. Research on the key technology of FMCW GPR, Chang'an University 2012, In Chinese

[4] Wang,Y.B Research on influencing factors of GPR detection accuracy, Technology Innovation Herald, 2011, (33).

[5] Gao,X. Research on processing and object recognition of Shallow formation's GPR signal. China Ocean University 2011. In Chinese

[6] Xie Z.H. 2005. Research on GPR image features of different target body underground. Engineering Geophysics, 1.

[7] Chen, Y.G, \& Xiao, X. The status quo and development on the ground penetrating radar status. Engineering Geophysics 2005, 2,149-155.

[8] Yang X.G. The choose of substation grounding system grounding resistance reduction programs and materials. North China Electric Power University, 2011. In Chinese 